\title{
DEVS based Network: Modeling and Simulation of Propagation Processes in a Multi-Layers Network
}

\author{
Youssef Bouanan, Gregory Zacharewicz, Bruno \\ Vallespir, Judicael Ribault \\ Univ. Bordeaux IMS UMR 5218 \\ F-33400 Talence, France. \\ youssef.bouanan@ims-bordeaux.fr \\ gregory.zacharewicz@ims-bordeaux.fr \\ bruno.vallespir@ims-bordeaux.fr \\ jribault@gmail.com
}

\author{
Saikou Y. Diallo \\ Virginia Modeling, Analysis and \\ Simulation Center \\ Old Dominion University \\ 1030 University Blvd. Suffolk, \\ VA 23435, USA. \\ sdiallo@odu.edu
}

\begin{abstract}
This paper deals with diffusion process in a multi-layer network using DEVS (Discrete Event System Specification) formalism. Social interaction plays an important role in studying the propagation of information, innovation, ideas, and influence among its members. When an informational event appears it can either die out quickly or makes significant inroads into a population. Network diffusion process allows us to understand the dynamics and the propagation of information in Social Network (SN). These networks can consist of individuals, group of person or organizations. The interactions could be done by physical proximity contact, remote collaboration, any types of social meetings and some forms of verbal or written communication depending on the situations. In our work we use the DEVS formalism, extended by DS-DEVS and PDEVS in order to set to simulate the propagation phenomena within a multi-layer social network (MSN). The network agent behavior is represented in the form of atomic models or coupled models if they are complex, and the social network is managed using DS-DEVS.
\end{abstract}

\section{Author Keywords}

Diffusion Process; Multi-Layer Network; DEVS formalism; Agent-Based Modeling;

\section{ACM Classification Keywords}

I.6.1 SIMULATION AND MODELING

\section{INTRODUCTION}

Many real-world systems can be modeled as networks, i.e., sets of interconnected entities. Several studies of networks employed an abstraction in which systems are represented as ordinary graphs [1]: the 'nodes' (or 'vertices') of the graphs represent some entity or agent, and a tie between a pair of nodes is represented using a single, static, unweighted 'edge' (or 'link'). Self- and multi-edges were also typically ignored. In real world, more than one kind of

SpringSim-MSCIAAS, 2016 April 3-6, Pasadena, CA, USA relationship can exist between two actors (e.g. family, friendship, religion and work ties) and that those ties can be so intertwined that it is impossible to analyze them separately $[2,3,4]$.

At the beginning such networks have been called multiplex network [5]. The term is derived from communications theory which defines multiplex as combining multiple signals into one in such way that it is possible to separate them if needed. Recently, the area of multi-layered social network has started attracting more and more attention in research conducted within different domains $[6,7,4]$, and the meaning of multiplex network has expanded and covers not only social relationships but any kind of connection, e.g. based on geography, occupation, kinship, hobbies, etc. [8].

Social interaction plays an important role in studying the propagation of information, innovation, ideas, and influence among its members. When an event appears - for example, the use of cell phones among students, the adoption of a new information system within the enterprise, or the rise of a political movement in an unstable society - it can either die out quickly or makes significant inroads into a population. Network diffusion process allows us to understand the dynamics and the propagation of information in social network. These networks can consist of individuals, group of person or organizations. The interactions could be done by physical contact, remote collaboration, any types of social meetings or some forms of verbal or written communication depending on the situations. The diffusion studies are numerous because diffusion phenomena are discussed in several disciplines: computer science (computer virus, information diffusion in a social network) [9], biology (epidemics) [10], physics, etc. In our case we diffuse a message enclosed in a data packet. This packet contains several pieces of information which influence the reaction of the individual and time life of the packet.

To this end, we analyze the topic of spreading processes in multilayer networks according to three main aspects: (A) 
how spreading processes can be modeled (Section 3), (B) what results can be obtained from these models (Section 4) and (C) how these results can be verified and validated (Section 5).This paper begins by introducing basic concepts (multilayer networks, spreading processes in multilayer networks and variables used to study these phenomena). Then, it provides model of individuals with DEVS characterized by a set of attributes and it presents our architecture to simulate the diffusion process in a multilayer network. The final part concerns our experiments and the conclusion.

\section{PRELIMINARIES}

In this section we introduce formal specification that can use to model and simulate a network. Then, we will present the concepts of multilayer network and dissemination processes in multilayer network.

In order to specify dynamical models, B.P. Zeigler introduced the Discrete EVent System Specification formalism (DEVS) [11]. With DEVS, a model of a large system can be decomposed into smaller component models with coupling specification between them. DEVS formalism defines two kinds of models: (1) atomic models that represent the basic models providing specifications for the dynamics of a sub-system using transition functions (external transition, internal transition); (2) coupled models or "network of models" that describe how to couple several component models together to form a new model. DEVS provides an automatic simulation based on time synchronization and message propagation.

In 1985, G. Klir was the first to introduce the concept of dynamic structures within a framework of modelling and simulation [12]. After, F. Barros introduced the DSDEVS formalism [13]. The latter constitutes the most satisfactory formalization for the modelling of dynamical changes in structure. Let us cite F. Barros writing about dynamic structure systems in general [13]: “[. . . ] Structural changes can better reflect dynamic of real systems we want to model in which drastic changes of structure and behaviors can be observed". This assertion is particularly relevant in the context of MAS where relations between agents are likely to change dynamically. Thus, DSDEVS is worth for the formalization of MAS in the context of dynamical systems. A simple network (i.e. a single-layer network) can be represented by a graph $[1,14]$. A graph is a tuple $G=(V$; $\mathrm{E}$ ), where $\mathrm{V}$ is set of nodes (vertices) representing social entities: humans, organizations, departments etc., called also members, agents or instances and $\mathrm{E} \subseteq \mathrm{V} \times \mathrm{V}$ is a set of (ordered or unordered) edges (arcs, connections or ties) that connects pair of nodes. Since social networks usually represent one kind of relationships they are also called single-layered social network SSN [15]. A multilayer network is a data structure made of multiple layers, where each layer is a monoplex network. A multi-layered social network (MSN) is a network extended to multiple edges between pairs of nodes/actors. It is defined as a tuple $<\mathrm{V}, \mathrm{E}$, L> where:

- $\mathrm{V}-$ is a set of actors (social entities);

- $\mathrm{E}$ - is a set of tuples $<\mathrm{v}, \mathrm{e}, \mathrm{l}>, \mathrm{l} \in \mathrm{L}, \mathrm{v} \neq \mathrm{e}$;

- $\mathrm{L}-$ is a set of distinct layers (types of relationships).

Each layer in the MSN corresponds to one type of relationships between people. Different relationships can result from the character of connections, types of communication channel. The examples of different relationships can be: friendship, family or work. Different communication channels that result in different types of connections are: email exchange, VoIP calls, instant messenger chats, etc. These networks can be defined as a sequence of graphs:

$$
\left\{\mathrm{G}_{\gamma}\right\}_{\gamma}{ }^{\delta}=\left\{\left(\mathrm{V}_{\gamma}, \mathrm{E}_{\gamma}\right)\right\}_{\gamma}{ }^{\delta}=1
$$

Where $\mathrm{E}_{\gamma} \subseteq \mathrm{V}_{\gamma} \times \mathrm{V}_{\gamma}$ is the set of edges and $\gamma$ indexes the graphs or the type of relationships. In our networks, the nodes (agents) are the same across the different layers (i.e. $\left.\mathrm{V}_{\gamma}=\mathrm{V}_{\delta} \forall \gamma, \delta\right)$.

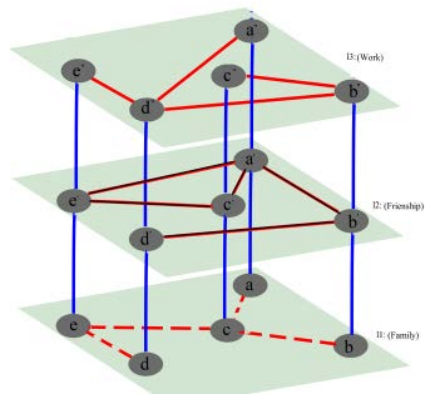

Figure 1. An example of the MSN.

In Figure 1, the example of three-layered social network is presented. The set of agents consists of $\{a, b, c, d, e\}$, they are connected with each other on three layers: $l_{1}$ (family), 12 (friendship) and 13 (work).

Diffusion processes in networks have a long history in social sciences [16]. With the advent of sufficient storage and computational power, this network diffusion process became an emerging research area in computer science [17]. Propagation models are designed to reproduce phenomena observed in social networks with applications in viral marketing, spread of disease and diffusion of ideas and innovations. Most models proposed recently are extensions from the independent cascade (IC) [18] and the linear threshold models (LT) [19]. The two models characterize two different aspects of social interaction. The IC model focuses on individual (and independent) interaction and influence among friends in a social network. The IC models can also be identified with the so-called 
Susceptible/Infective/Recovered (SIR) model for the spread of disease in a network [20]. The LT model focuses on the threshold behavior in influence propagation, which we can frequently relate to; when enough of our friends bought a new phone, played a new computer game, or used new online social networks, we may be converted to follow the same action.

One of the important ideas in the context of dissemination processes in multilayer networks is the fact that elements can also spread from one layer to another. In general there are four possibilities for an element to traverse a multilayer network (Table 1): same-node inter-layer, when the cascade switches layer but remains on the same node, e.g., when an element is shared on different layers by the same actor; other-node inter-layer, when a cascade continues spreading to another node in another layer. In third type, other-node intra-layer, the cascade continues spreading through the same layer, e.g., spreading an information between friends. The fourth combination, same-node intralayer, is generally not considered meaningful and therefore omitted in all the diffusion studies we have considered.

\begin{tabular}{|c|c|c|}
\hline & Same Node & Other Node \\
\hline $\begin{array}{c}\text { Same } \\
\text { Layer }\end{array}$ & Unused & $\begin{array}{c}\text { The item spreads to } \\
\text { a different node on } \\
\text { the same layer }\end{array}$ \\
\hline $\begin{array}{c}\text { Other } \\
\text { Layer }\end{array}$ & $\begin{array}{l}\text { The item switches } \\
\text { layer but remains } \\
\text { on the same node }\end{array}$ & $\begin{array}{c}\text { The element } \\
\text { continues spreading } \\
\text { in a different node } \\
\text { on the another layer }\end{array}$ \\
\hline
\end{tabular}

Table 1. Different possibilities for spreading an element in a multilayer network.

\section{MATERIALS AND METHODS}

In this section, we propose an agent-based model for the information diffusion in a Multi-layer network.

\subsection{Problem Statement}

Several research studies attempted to model and simulate the influence diffusion in a social network. However, the focus is on one layer network (one link or relationship between persons) e.g., the message disseminates only within friends. In addition, they did not consider the individual and cultural factors on diffusion process; the message disseminates in the same way between two friends or two coworkers. Actually, the way how people communicate is highly dependent of the person who people are talking to. In the current work, we address the following issue: how to simulate the effects of information on population with a propagation algorithm of the effects across Multi-Layer social networks? The challenge is to improve the realism of socially intelligent agents and take into account the impact of individual's personality and cultural values in the diffusion model process.

\subsection{Formalization of Human Behavior}

In the agent-based model, individuals or group of individuals are represented as agents. Each agent is described by a set of attributes:

- Static attributes: gender, social status, religion, age class, ethnicity, leadership and language.

- Dynamic attributes (variables): opinion, interest, un/satisfied-needs.

Static attributes are intrinsic or unchanged parameters, i.e., time has no effect on them. Dynamic attributes evolve with time or events. For example, individuals can be reached or not by the information depending on its opinion and the social network configuration.

We use DEVS to describe the human behavior. DEVS is a well-defined formalism which has numerous advantages over other formalism in the modeling of complex dynamic system. The perception is specified by the arrival of external events on the model's ports. These events change the state of the agent. We can represent the actions of agent by a discrete event generated by the agent, i.e., the emission of an external event by an output function. In a simplified way, we can represent the agent's autonomy as the union of internal transitions of a DEVS model. Finally, Perception, action and autonomy formalize the behavior of the agent.

The following notations describe the server model. We do not describe detailed operations of this model; we just explain the role and interface of the server model (ServerNode).

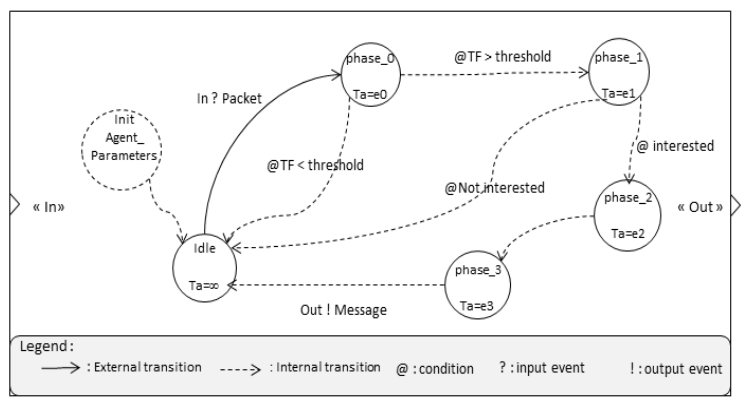

Figure 2. DEVS specification of agent model.

Figure 2 is an example that describes the message influence on the individual behavior and potentially its dissemination using the DEVS specifications. The first phase is used to configure and initialize the agent' attributes. Then, when the agent is in the "IDLE" phase, and if it receives an external event from another agent on port "In" (In? Packet), it moves to "phase_0". Here, the agent calculates the trust factor $\alpha(\mathrm{i}, \mathrm{j})$ between him and the sender depending on the social pressure, similarity between him and the sender 
(based on the religion, language and age class) and its sensitivity to the message theme. If the trust factor is higher than a predefined threshold, the receiver moves to "phase 1" else it returns to "IDLE". Then, if the message is still strong enough, the receiver moves to "phase_2" else it returns to "IDLE". This message creates an impact on the individual, and eventually its behavior depending on the agent's opinion and the relationship between him and the sender. After a period, processing time, the receiver transmits the message on its ego-network. After the contact between receiver and sender, the receiver's variables (opinion, interest, satisfaction) change according to the message content, the sender and cultural factors.

\subsection{Formal Specification of Social Network}

As recalled previously, DEVS formalism is supporting describing static coupled models (or graphs). However, the relations between agents are able to change dynamically, requires a dynamic structure. Works to introduce this type of structure was proposed $[13,21]$ to be integrated in DEVS. The formalization of F. Barros called DS-DEVS for Dynamic-Structure DEVS, is more satisfactory in the case of social networks M\&S since it preserves all the properties of DEVS, i.e. the modularity and the hierarchical decomposition. DS-DEVS is represented in the form of a coupled model thus which can couple itself with other models DEVS. It makes it possible to modify connections between its models and to add or remove its DEVS models at simulation run time on the occurrence of internal or external events.

We created a tool for the automatic instantiation of individuals attributes in behavioral DEVS models and relations between them (static $\mathrm{SN}$ graph from social science literature classical structure) to coupled models managed using DS-DEVS. The agents are instantiated in the form of atomic models or if they have a complex dynamics in coupled models (as described in Figure 3). DEVS allows here the possibility of defining dynamically each entity and simulating the evolution of the system.

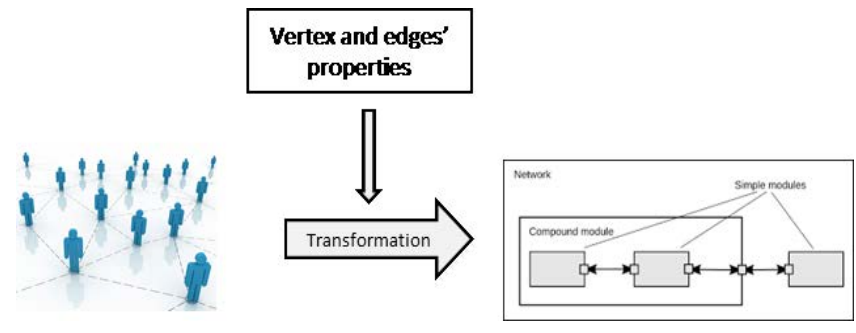

Figure 3. Specification of a Network.

\subsection{Modeling and Simulating Dissemination Process in a Multi-Layer Network}

In this work, we use an extension of social influence model called "User-centered model". Each behavioral model includes (a) social pressure, measured from the number of active neighbors, (b) the sensibility of the actor to the message or the item disseminated and (c) the similarity between the sender and the receiver of the item (Figure 4). Then according to rules the information is touching the agent or not.

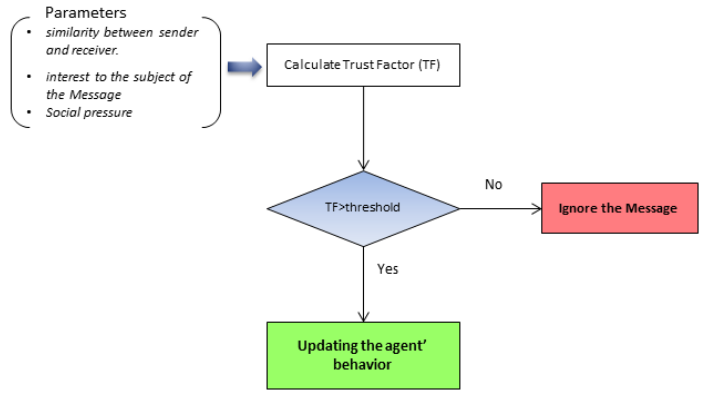

Figure 4. Diffusion algorithm between agents.

Then, at the structural level, flattening different layers into a single network was one possible way to simulate multilayer network. In this flattened network, individuals are connected to other individuals directly. Benefits are the simplicity of representation and the strict minimum number of components and bindings. One drawback is the nonseparation of concerns.

Another barrier faced has been that each individual must contain concern (as an atomic or as a coupled model) about the network rules (to transmit or not the information on this specific network for example) as well as individual rules (affected or not by the message for example). This nonseparation of concern prevents us to reuse the model with other networks or in other study.

To overcome these two barriers, we presented a novel architecture described in Figure 5. We want to keep the separation of concerns at the network level, each network remains independant from the others. component $a, a$, and $a$ ' are called Proxy and contains the specific network rules for each individual. Each component has a specific behavior depending on the type of the link connecting it to the ego-network. These components are connected to a Server representing the individual state and containing the individual rules. The sequence when an information arrive to $a$ "' on Networkl is the following :

- component $a$ "’ sends an event to Server A

- Server A reads the event and depending on its state (motivation) and rules, can propagate the information to its networks. Thus, an event is sent to components $a, a$ ' and $a$ '”.

- Components $a, a$, and $a$ "' read the event and depending on their state and rules, can diffuse the information to their neighbors. In this case $a$ " already has the information and does nothing; $a^{\prime}$ sends an event to $b^{\prime}$ and $d^{\prime}$; $a$ sends an event to $b$. 
- components $b, b^{\prime}$ and $d^{\prime}$ send an event to respectively proxy $B$ and $D$ and so on.

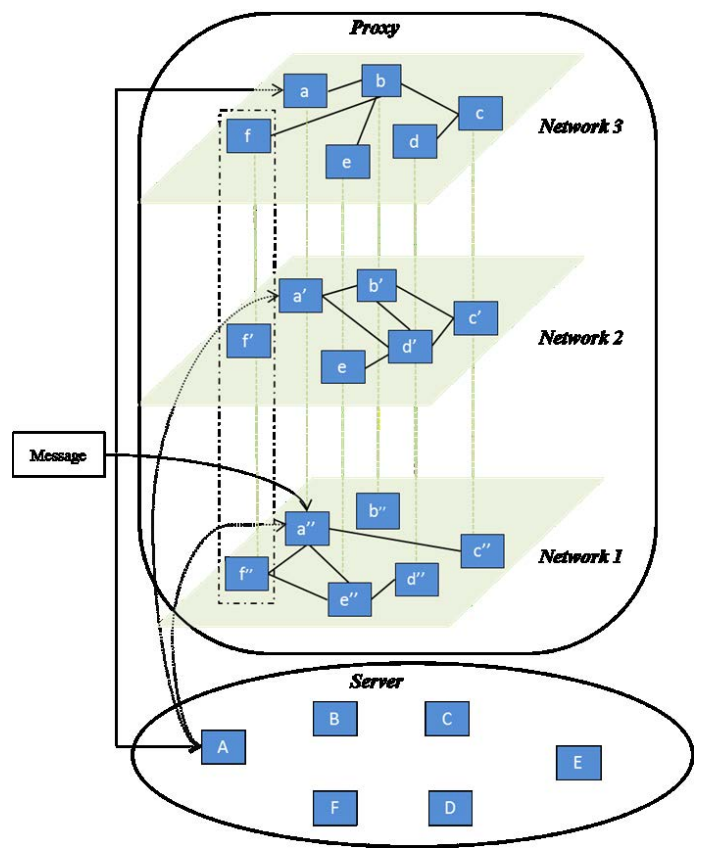

Figure 5. Proposed MSN implementation.

In our case we implemented the diffusion model presented in the section 3.4 in the proxies and the server updates the agents behavior if it receives an notification from the proxies (the proxy has an interest to the item). This architecture allows us the integration of various diffusion model taking into account the caracteristics of the layer (type of relationship or the communication channel). This approach aims to enhance the reusability, the model representation, and thus eases the development. It's easy to add a new network: we just have to build the specific rules (if any) in a new Proxy component. We can change individuals without changing the networks. Drawbacks are the increased number of nodes and bindings.

Four conditions can cause the end of the simulation: (1) The individual who receives the information does not have an interest; (2) The strength of the message to be propagated falls below a given threshold; (3) All nodes are inactive; (4) It has been a long time (higher than a given threshold) since the scenario occurred.

Pre-simulation: Before making a simulation, we build a new experiment by generating a multi-relational graph if it does not already exist in the repository. An experiment includes one or several groups. A group includes many individuals. A graph represents all relations between individuals. We opted for the storage of information in a database so that each experiment is easily accessible and replayable. The repository contains all the models available to execute the simulations, i.e., all the servers and proxies that will be used by the Graph-Executive to produce the simulation model.
Simulation: The agent-based model is used to simulate the process of items diffusion in the network generated in the first step. The simulation starts with the experiment to simulate, as well as servers and proxies to use. In this way, for the same experiment, we can test different behavior algorithms. The Graph-Executive connects to the database to retrieve all the information from the experience to execute. It transforms the graph into a DEVS coupled model "DEVS network". Each node is transformed to a DEVS atomic model. The generator is used to prepare the item and send it to info-sources at time $\mathrm{t}=0$. The simulation is executed using to produces a result set in the form of a file. This part will be developed within the framework of VLE application (Virtual Laboratory Environment) [22].

Post-simulation: The $\mathrm{R}$ script post-processes the simulation result. The result file is used to visualize the course of the simulation and to develop an analysis and conclusion. The analysis can lead to a new simulation, i.e., makes a new pre-simulation, simulation and postsimulation.

\section{EXPERIMENT}

\subsection{Overview}

The MSN concept introduced in this paper is illustrated by modeling and simulating the spread of information in a multi-layer social network. The goal of this experiment is to study the diffusion phenomena in the framework of multilayers networks. The analyze and study of the information influence processes initiated by starting point over network member attributes is done regarding temporal and sequential dynamic. Simulation is used to show that the topology of the network can affect the result of the diffusion process. In more details, a population of 350 agents has been generated according to social science population structure rules described in [23] and connected on three layers: family, friends and neighbors. These three layers represent the primary groups defined in [24]. Assuming that all agents are starting in a state " 0 " (they don't received the message yet), and when an agent receives the message, its state changes to " 1 " if it has an interest to the message or changes to " 2 " if it receives the message but it has no interest to the message or the item. The agents in the state " 2 " block the spreading process and they do not update their behavior. However, agents in state " 1 " update their behavior and their parameters. In this case, the update of agent's opinion is based on the concept of opinion change described by [25]. Social influence occurs when a message emitter and a message receiver get in contact. The message emitter attempts to communicate position about an opinion to the receiver. As result of this conversation, the receiver of the message may shift her/his opinion some distance towards or away from that of the emitter's opinion. This opinion is characterized by the opinion number and the opinion confidence bounds. 


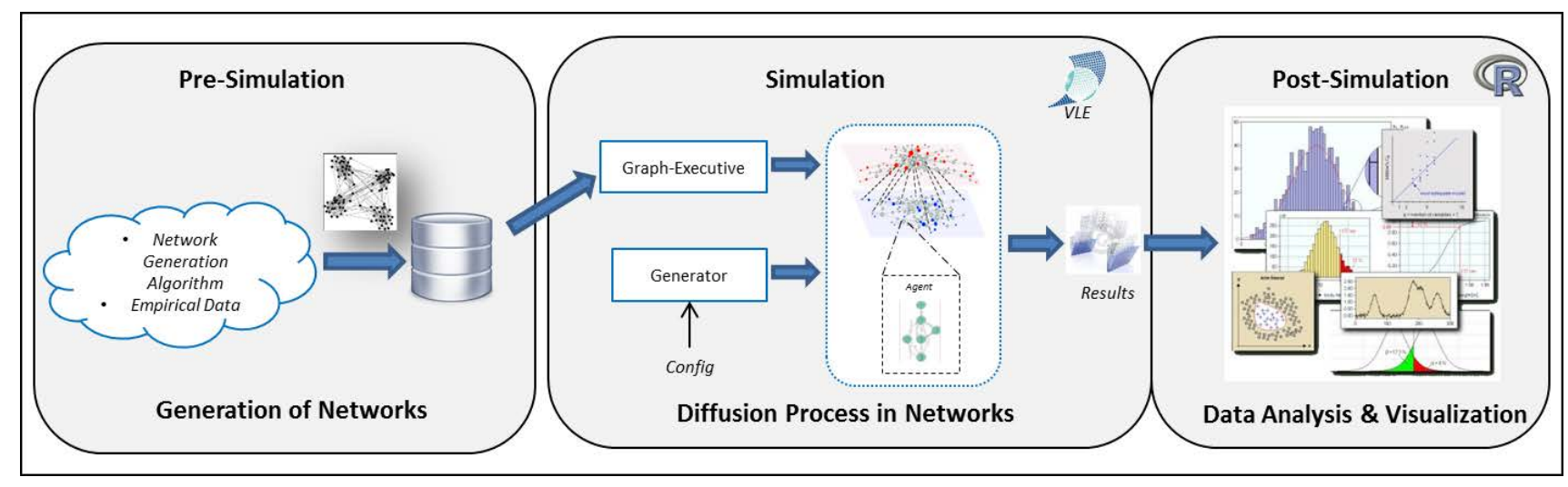

Figure 6. Agent-Based Simulation Architecture.

The study proposed to distinguish two categories of population: extremist and moderate people. The first category is located at the ends of the distribution of opinion (their uncertainty is lower, they block the messages).

\subsection{Settings}

We have driven in the experiment proposed two scenarios with the same population characteristics but with different configurations for the information spreading settings.

Scenario 1: The goal of this scenario is to show the impact of agent's attributes and message characteristics on diffusion phenomenon. Therefore, we ply 40 simulations by connecting the generator (at time $\mathrm{t}=0$ ) to the political leaders and changing randomly the topic of the message and its strength. Each layer has a different sensitivity to the theme of the message. We propose three themes: (1) message affects security and stability in the area; (2) message affects the religious community; and (3) it influence physical and social needs: health and employment. Then, we analyze these results to verify and validate the different hypotheses.

Scenario 2: In this experiment, we fix all parameters in the model including the MSN structure, agent settings and message content and strength. Only the message starting point will be a changed. Before starting simulation, we connect the generator with three different agents (infosources). We change these points randomly for each simulation. In the end we store the result of 50 simulations in a csv file. After that, we analyze the variation of the final opinion depending on different combinations of starting point.

\subsection{Result}

Figure 7 presents outputs from scenario 1 . It presents the average of agent's opinion for each simulation depending on message characteristics. For each iteration the strength of the message has been changed randomly between $[1,10]$ and the subject of the message between 1 and 4 . These four message subjects influence the diffusion of the message according to each dimension.

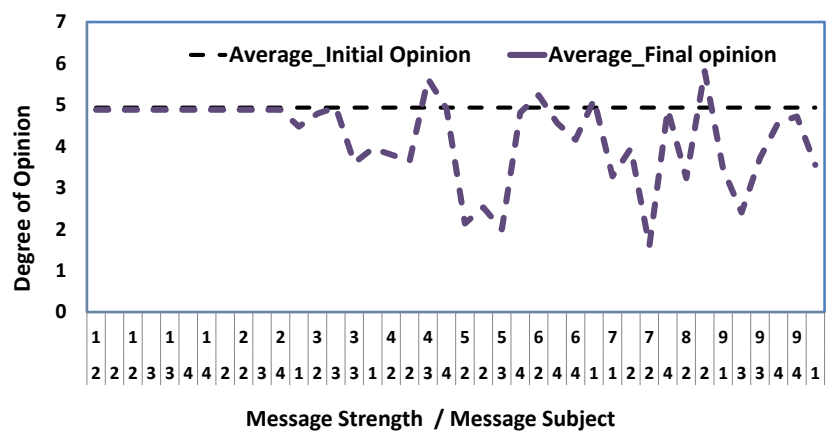

Figure 7. The final opinion of agents relative to the message characteristics.

The payoff (efficiency) of the actions is affected by the message characteristics and the strengths of a message. The objective is to reveal how each agent is impacted over time by a message. Figure 7 shows that the messages spread in this example have majorly a negative impact on the opinion of the individuals regarding a given event. It is displayed by an average final opinion (value $<5$ ) mostly under the average initial opinion (value $=5$ ). Nevertheless it distinguishes that some couple of (message/content and message/strength) have a specific significant impact over a population.

At this level, statistical test are helpful for the validation of this work. Nevertheless even a simple analysis of the result reveals by simulation the possibility to maximize the effect of the message over a population. It can be done by giving the right strength and content value to the message (it can be adapted by tuning over replications of simulation run) in order to optimize the effects over the population and to control the effort regarding potential results. 


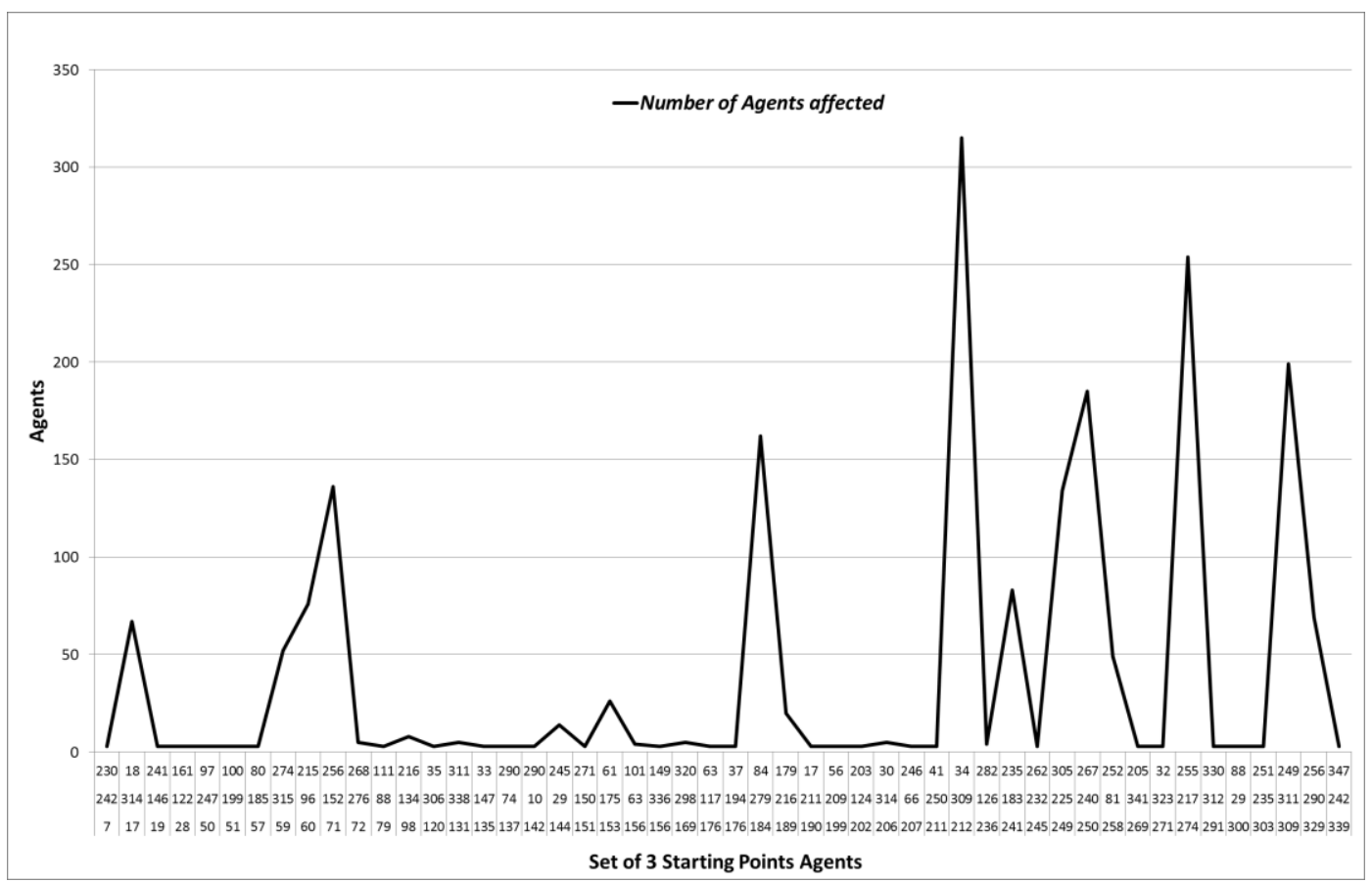

Figure 8. Number of agents affected relative to different info-sources.

Figure 8 presents outputs of scenario 2. It shows the number of agent interested by the message during each iteration. For each iteration, three starting points have been randomly changed. This Figure permits to observe significant impact or insignificant effects. On one hand, several combinations do not trigger the propagation process for several reasons. On the other hand for instance one combination has a deep impact on the population reaching a pick of a bit less than 330 individuals. The reason of the success/fail can be depending on the info-sources egonetworks or the rejection of the messages by the receivers along the chain of diffusion process. This example remains conceptual. Then, domain dependent thresholds can be defined to set the significance barriers.

The conclusion of this experiment is that the topology or structure of a network is very important in diffusion phenomenon and even if the Node has a high eigenvalue or a good centrality the reach of the actions can be insignificant. Conversely, some starting points that were not identified as key agents (such as not leader and some sometime not well interconnected) can be revealed by simulation as key path to maximize the information diffusion (some picked in the Figure 8 are not leaders and not connected to important number of neighbors).

In this paper, we focused on statistical test in order to verify our hypothesis and to analyze the sensitivity of the simulation models. In the next step, we will adopt others verification and validation techniques of simulation models (e.g. comparing the results of our simulation with historical data).

\section{CONCLUSION AND PERSPECTIVE}

This paper presents the architecture of a multidimensional social network for modeling relationships between people. It uses an MSN architecture to generate each dimension with its own rules: the friendship dimension is different from the neighbor dimension and cannot be generated in the same way. So, using an MSN has permitted a better partitioning of each social dimension of human life. Furthermore MSN also allows defining distinct propagation rules for each dimension. The MSN environment implemented proposes features to easily modify such as the strength of the message, the time of the day, the probability of acceptance for each dimension. The simulation has permitted to obtain first results to observe the impact of the population structure and behavior in the message diffusion process. The simulation of two scenarios has presented the interest of simulation to identify key info source in the population and the importance of the tuning over the message strength and content to maximize its impact on the population. One of the greatest things using an MSN is its adaptability. The tool allows easily adding or deleting a dimension in respect of the population's features.

Finally, the work presented in this paper is part of a defense related project but opens many possibilities for various application domains. Generating a population with an unseen level of cultural features can be used in marketing to simulate the adoption of a new product, in social science to observe dynamically the diffusion of information or the way an opinion changes. 


\section{ACKNOWLEDGMENTS}

This work is supported by the SICOMORES Project $\mathrm{N}^{\circ}$ 132936073 funded by French DGA (Direction Générale de l'Armement) and MASA Group. It involves the following partners: IMS university of Bordeaux, LSIS university of Marseille and MASA Group.

\section{REFERENCES}

1. Bollobás, B. Modern Graph Theory. Berlin, Springer (1998).

2. Fienberg S. E., Meyer M. M., Wasserman S.S., Statistical Analysis of Multiple Sociometric Relations. Journal of American Statistical Association, 80. 389 (1985): 51-67.

3. Minor, Michael J. New directions in multiplexity analysis. Applied network analysis: A methodological introduction (1983): 223-244.

4. Szell, Michael, Renaud Lambiotte, and Stefan Thurner. Multirelational organization of large-scale social networks in an online world. Proceedings of the National Academy of Sciences 107.31 (2010): 1363613641 .

5. Monge, Peter R., and Noshir S. Contractor. Theories of communication networks. Oxford University Press, 2003.

6. Kazienko, Przemysław, Katarzyna Musiał, and Tomasz Kajdanowicz. Multidimensional social network in the social recommender system. Systems, Man and Cybernetics, Part A: Systems and Humans, IEEE Transactions on 41.4 (2011): 746-759.

7. Rodriguez, Marko A., and Joshua Shinavier. Exposing multi-relational networks to single-relational network analysis algorithms. Journal of Informetrics 4.1 (2010): $29-41$.

8. Abraham, Ittai, et al. Low-distortion inference of latent similarities from a multiplex social network. Proceedings of the Twenty-Fourth Annual ACM-SIAM Symposium on Discrete Algorithms. SIAM, 2013.

9. Girvan, Michelle, et al. Simple model of epidemics with pathogen mutation. Physical Review E 65.3 (2002): 031915 .

10. Cauchemez, Simon, et al. Role of social networks in shaping disease transmission during a community outbreak of $2009 \mathrm{H} 1 \mathrm{~N} 1$ pandemic influenza. Proceedings of the National Academy of Sciences 108.7 (2011): 2825-2830.

11.Zeigler, Bernard P., Herbert Praehofer, and Tag Gon Kim. Theory of modeling and simulation: integrating discrete event and continuous complex dynamic systems. Academic press, 2000.

12. Klir, George. Architecture of systems problem solving. Springer Science \& Business Media, 2013.
13. Barros, Fernando J. Modeling formalisms for dynamic structure systems. ACM Transactions on Modeling and Computer Simulation (TOMACS) 7.4 (1997): 501-515.

14. Newman, Mark. Networks: an introduction. Oxford University Press, 2010.

15. Magnani, Matteo, and Luca Rossi. The ML-model for multi-layer social networks. Advances in Social Networks Analysis and Mining (ASONAM), 2011 International Conference on. IEEE, 2011.

16. Rogers, E. M. Diffusion of innovativeness. NY: The Free Press of Glencoe(1962).

17. Domingos, Pedro. Mining social networks for viral marketing. IEEE Intelligent Systems 20.1 (2005): 80-82.

18. Goldenberg, Jacob, Barak Libai, and Eitan Muller. Talk of the network: A complex systems look at the underlying process of word-of-mouth. Marketing letters 12.3 (2001): 211-223.

19. Granovetter, M. Threshold models of collective behavior. American journal of sociology, (1978): 14201443.

20. Bailey, N. T. (1975). The mathematical theory of infectious diseases and its applications. Charles Griffin \& Company Ltd, 5a Crendon Street, High Wycombe, Bucks HP13 6LE.

21. Uhrmacher, Adelinde M. Dynamic structures in modeling and simulation: a reflective approach. ACM Transactions on Modeling and Computer Simulation (TOMACS) 11.2 (2001): 206-232.

22. Gauthier Quesnel, Raphaël Duboz, Éric Ramat, The Virtual Laboratory Environment - An operational framework for multi-modelling, simulation and analysis of complex dynamical systems, Simulation Modelling Practice and Theory, Volume 17, Issue 4, April 2009, Pages 641-653, ISSN 1569-190X.

23. Forestier, Mathilde, et al. "Generating Multidimensional Social Network to Simulate the Propagation of Information." Proceedings of the 2015 IEEE/ACM International Conference on Advances in Social Networks Analysis and Mining 2015. ACM, 2015.

24. Litwak, Eugene, and Ivan Szelenyi. Primary group structures and their functions: Kin, neighbors, and friends. American Sociological Review (1969): 465481.

25. Friedkin, Noah E., and Eugene C. Johnsen. Social influence networks and opinion change. Advances in group processes 16.1 (1999): 1-29. 